\title{
Living- donor liver transplantation with inferior vena cava replacement in an infant recipient with advanced hepatoblastoma
}

\author{
Jung-Man Namgoong ${ }^{1}$, Shin Hwang ${ }^{2}$, Seak Hee $\mathrm{Oh}^{3}$, Kyung Mo Kim ${ }^{3}$, Gil-Chun Park ${ }^{2}$, \\ Chul-Soo Ahn ${ }^{2}$, Hyunhee Kwon ${ }^{1}$, Yu Jeong $\mathrm{Cho}^{1}$, and Yong Jae Kwon ${ }^{1}$ \\ ${ }^{1}$ Division of Pediatric Surgery, Department of Surgery, Asan Medical Center, University of Ulsan College of \\ Medicine, ${ }^{2}$ Division of Hepatobiliary Surgery and Liver Transplantation, Department of Surgery, \\ Asan Medical Center, University of Ulsan College of Medicine, ${ }^{3}$ Department of Pediatrics, Asan Medical Center, \\ University of Ulsan College of Medicine, Seoul, Korea
}

\begin{abstract}
Replacement of the inferior vena cava (IVC) after concurrent resection of hepatoblastoma-containing liver and retrohepatic IVC is regarded as a feasible option for pediatric living-donor liver transplantation (LDLT). This technique makes the extent of resection of LDLT comparable to that of deceased-donor liver transplantation (DDLT). We present one case of pediatric LDLT with IVC homograft replacement for advanced hepatoblastoma. The patient was a 10-kg 18-month-old girl suffering from large multiple hepatoblastomas, which were partially regressed by neoadjuvant chemotherapy. Because the tumors had invaded the retrohepatic IVC, there was a high risk of residual tumor cells at the IVC if it was preserved. Thus, we decided to replace the IVC during the LDLT operation. After a cold-stored fresh iliac vein homograft was prepared, we performed LDLT using her mother's left lateral section liver graft. A 4-cm-long common iliac vein homograft was attached to the liver graft at the back table. The left lateral-section graft with IVC attachment was implanted using the standard procedures like those of DDLT. We also did portal vein graft interposition. The patient recovered uneventfully and has been undergoing scheduled adjuvant chemotherapy to date. This is our second case of IVC homograft replacement for pediatric LDLT. In pediatric recipients, various vein homografts, such as iliac vein, IVC, and other large veins, can be used depending on the body size of the recipient and availability of vein homografts. (Ann Hepatobiliary Pancreat Surg 2020;24:72-77)
\end{abstract}

Key Words: Interposition; Venoplasty; Pediatric transplantation

\section{INTRODUCTION}

Replacement of the inferior vena cava (IVC) after concurrent resection of hepatoblastoma-containing liver and retrohepatic IVC is an established optional technique of pediatric living-donor liver transplantation (LDLT). ${ }^{1-3}$ This technique converts the extent of hepatectomy for LDLT to that of deceased-donor liver transplantation (DDLT). Considering the ongoing physical growth of pediatric recipients, the only material usable for IVC replacement is a homologous vein graft. We previously presented a case of pediatric LDLT with IVC replacement using an IVC homograft. ${ }^{1}$ We herein present one case of IVC replacement with a common iliac vein homograft performed dur- ing LDLT operation in an infant patient with advanced hepatoblastoma.

\section{CASE}

The patient was an 18-month-old 10-kg girl suffering from large multiple hepatoblastomas. At 13 months after birth, abdominal distension was detected, and workup studies confirmed the diagnosis of hepatoblastoma (Fig. 1A, B). She underwent systemic chemotherapy, and the tumor size was gradually reduced, but some parts of the tumors remained, being partially responsive to systemic chemotherapy (Fig. 1C, D). Thus, we decided to perform LDLT to remove the tumor completely.

Received: January 30, 2020; Revised: February 3, 2020; Accepted: February 9, 2020

Corresponding author: Shin Hwang

Department of Surgery, Asan Medical Center, University of Ulsan College of Medicine, 88 Olympic-ro 43-gil, Songpa-gu, Seoul 05505, Korea Tel: +82-2-3010-3930, Fax: +82-2-3010-6701, E-mail: shwang@amc.seoul.kr

Copyright (C) 2020 by The Korean Association of Hepato-Biliary-Pancreatic Surgery

This is an Open Access article distributed under the terms of the Creative Commons Attribution Non-Commercial License (http://creativecommons.org/ censes/by-nc/4.0) which permits unrestricted non-commercial use, distribution, and reproduction in any medium, provided the original work is properly cited. Annals of Hepato-Biliary-Pancreatic Surgery • pISSN: 2508-5778 - elSSN: 2508-5859 

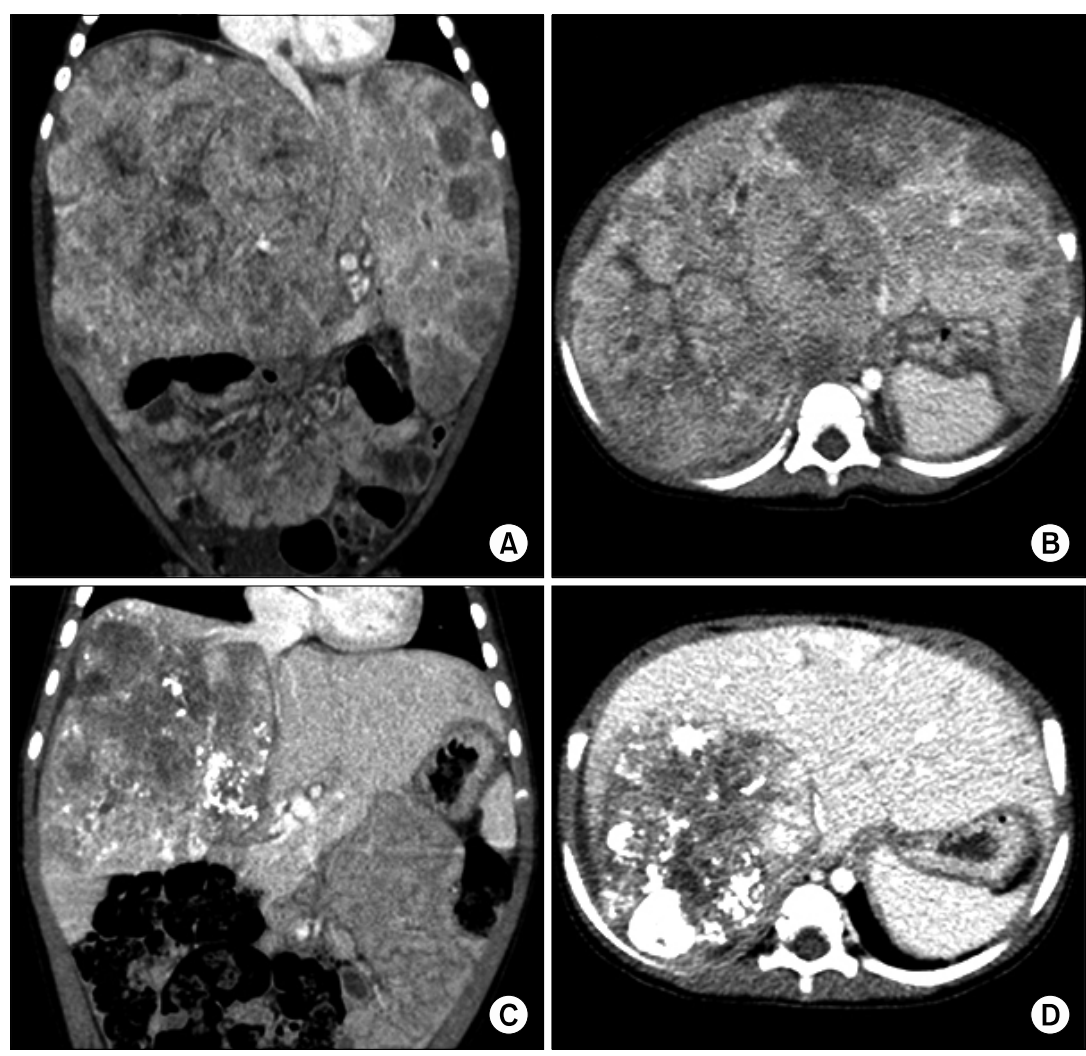

Fig. 1. Pretransplant computed tomography findings. At five months before the liver transplantation operation, huge multiple tumors occupied the whole liver, and the abdomen was distended by the enlarged liver (A and $\mathrm{B})$. At one month before liver transplantation, the tumor size was markedly regressed $(\mathrm{C}$ and D), but the retrohepatic inferior vena cava was invaded by the tumors $(\mathrm{C})$.
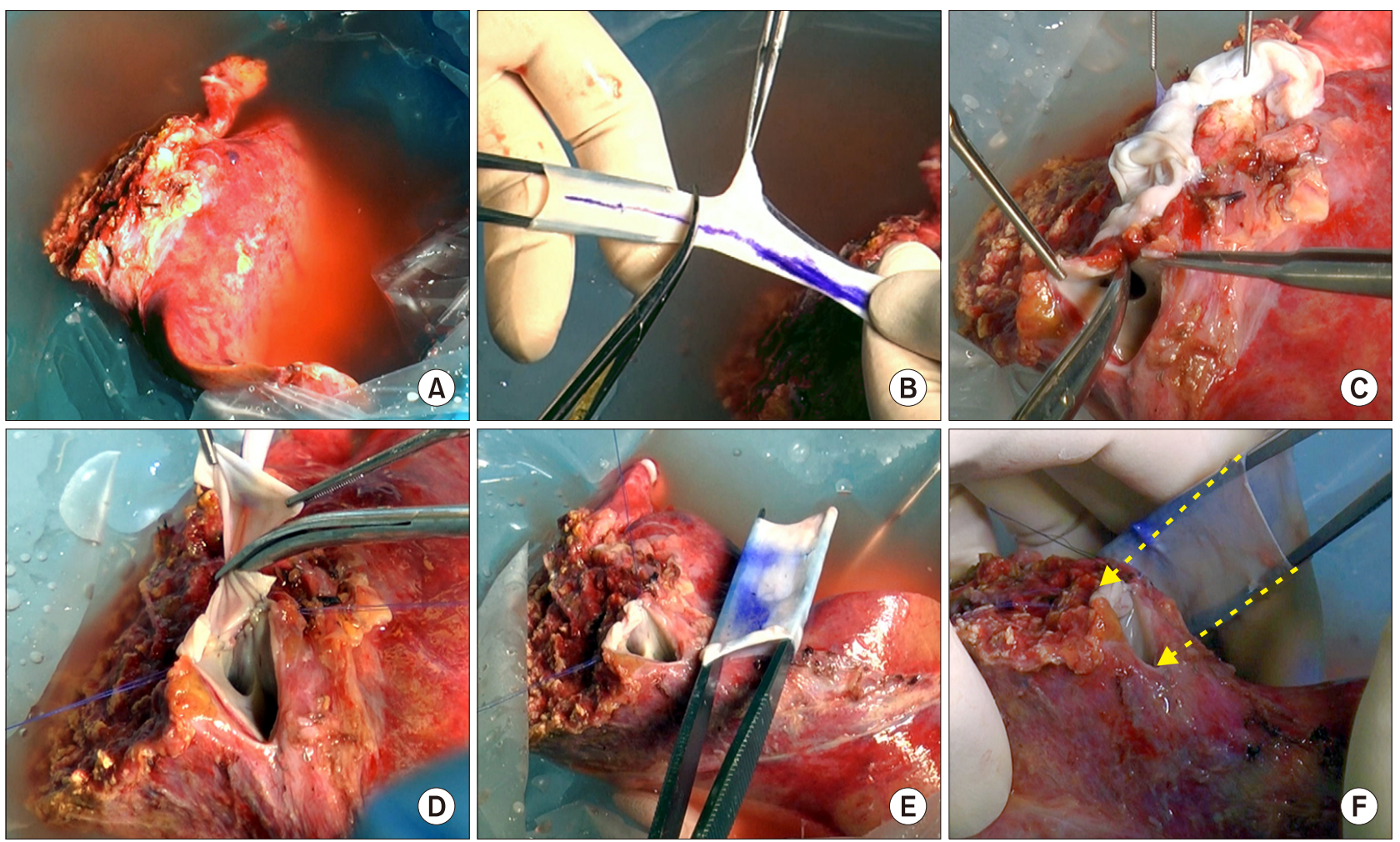

Fig. 2. Intraoperative photographs of the bench work. (A) A left lateral section graft was harvested from her mother. (B) The iliac-vein homograft was divided into two parts as the common iliac vein portion and external iliac-vein portion. (C) The graft hepatic vein orifice was incised for venoplasty. (D) An iliac vein patch was applied to the incised defect. (E) The sizes of the enlarged graft hepatic vein orifice and common iliac vein homograft were matched. (F) The 4-cm-long common iliac vein homograft was attached to replace the retrohepatic inferior vena cava (arrows). 

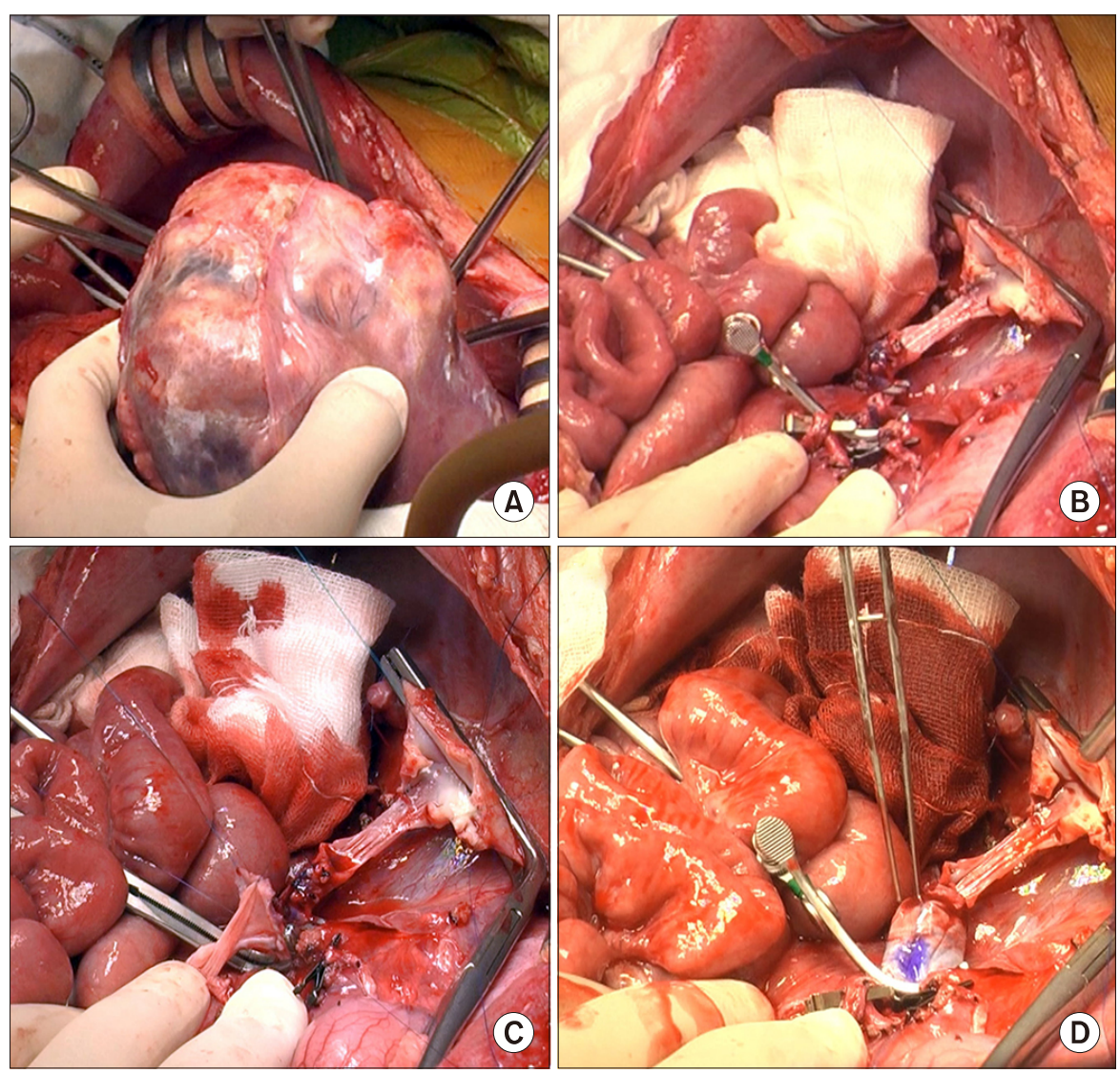

Fig. 3. Intraoperative photographs of the recipient hepatectomy and portal vein interposition venoplasty. (A) The recipient's native liver was removed. (B) Some part of the retrohepatic vein wall remained. (C) Portal vein venoplasty was done with interposition of an external iliac vein homograft. (D) The reconstructed portal vein was temporarily distended to check the security of anastomosis.
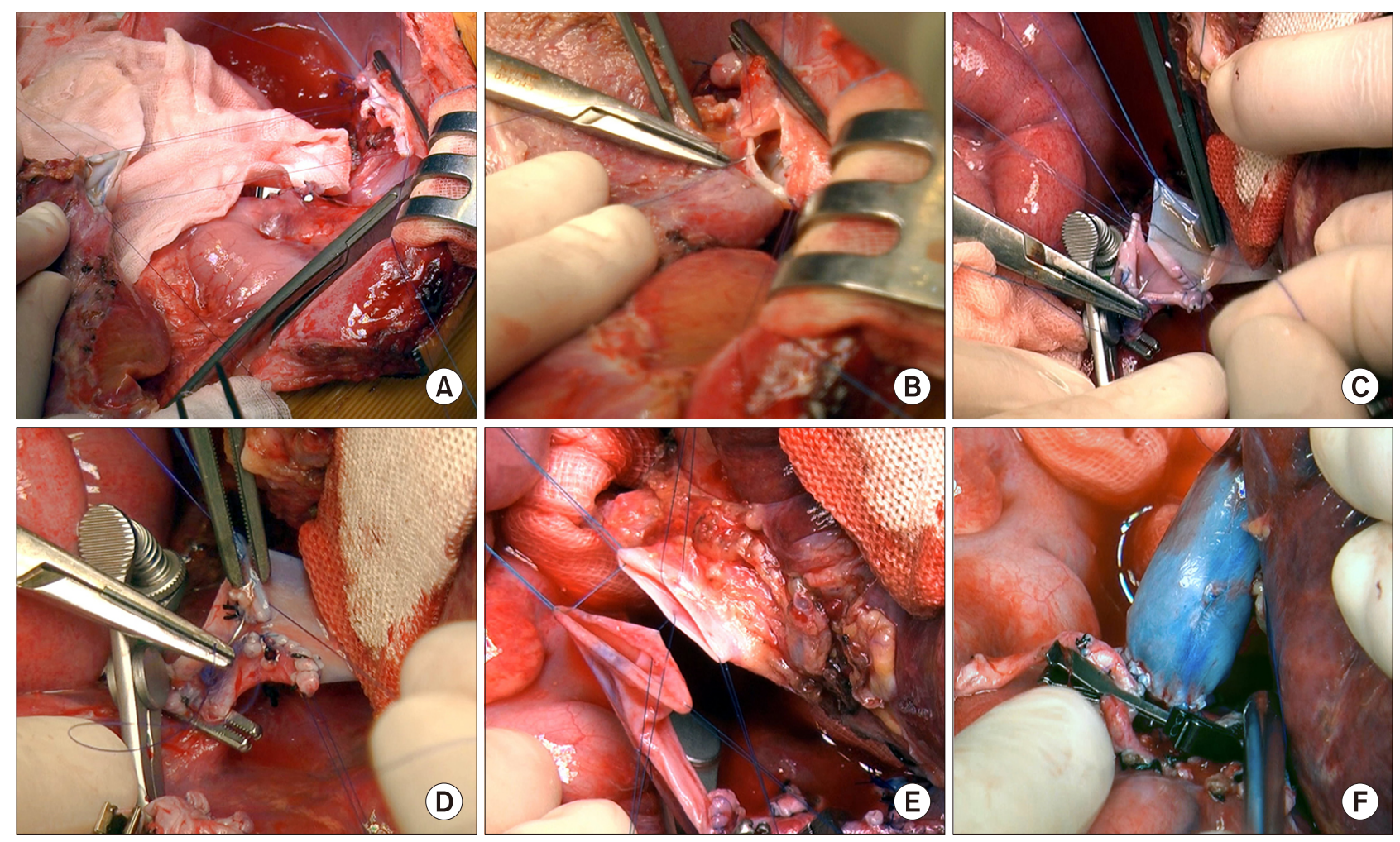

Fig. 4. Intraoperative photographs of the graft implantation. (A and B) The suprahepatic end of the vena cava was anastomosed to the graft hepatic veins. (C and D) The infrahepatic end of the vena cava was anastomosed to the interposed vein homograft after size matching. (E and F) The graft portal vein was anastomosed with the interposed external iliac vein homograft. 
The tumors encroached the retrohepatic IVC (Fig. 1C); thus we decided to replace the IVC during the LDLT operation. After we prepared a cold-stored fresh iliac homograft, which was recovered from a deceased organ donor, we performed the LDLT operation shortly after the living-donor workup.

A 220-g left lateral section liver graft was harvested from her 37-year-old mother (Fig. 2A). The diameter of the recipient's native retrohepatic IVC was $12-14 \mathrm{~mm}$ and that of the common iliac vein homograft was approximately $18 \mathrm{~mm}$; thus we unified the common iliac vein homograft with the graft hepatic vein orifice to make a conjoined opening as in the normal whole liver graft, instead of implanting the graft hepatic vein in the iliac vein homograft in an end-to-side fashion. The iliac vein homograft included the common iliac vein portion and external iliac vein portion; so the 4-cm-long common iliac vein portion was first excised to use for IVC replacement (Fig. 2B). The remaining portion of the homograft was used for a hepatic vein patch and portal vein interposition. The graft hepatic vein orifice was incised (Fig. 2C) and an iliac vein patch was applied to widen the orifice (Fig. 2D). After size matching (Fig. 2E), the common iliac vein homograft was attached to replace the retrohepatic IVC (Fig. 2F).

During right liver mobilization, the tumor-invaded right diaphragm was excised and repaired primarily. The recipient's native liver, including the anterior portion of the retrohepatic IVC, was removed (Fig. 3A, B). The recipient portal vein was enlarged by interposition of the external iliac vein homograft (Fig. 3C, D). The double-lumen outflow orifice of the left lateral section graft with IVC interposition was anastomosed with the recipient's widened suprahepatic IVC stump (Fig. 4A, B). The infrahepatic IVC stump was anastomosed with the interposed iliac vein homograft after length matching (Fig. 4C, D). The graft portal vein was anastomosed with the interposed external iliac vein homograft (Fig. 4E, F). One left hepatic artery was reconstructed, and Roux-en-Y hepaticojejunostomy was done. Since the liver graft was not large for the size of the recipient abdomen, the abdominal wall was repaired primarily.

The resected liver specimen showed multiple viable hepatoblastomas without lymph-node metastasis (Fig. 5). No vascular complication developed after LDLT operation
(Fig. 6). This patient recovered uneventfully (Fig. 7), and has been doing well for six months to date. She has been undergoing scheduled adjuvant chemotherapy.

\section{DISCUSSION}

Compared with DDLT, LDLT has a definite oncological demerit in patients with hepatic malignancy, because the recipient IVC should be preserved. If the IVC can be removed concurrently during LDLT operation, the extent of recipient hepatectomy becomes comparable to that of DDLT. Unlike adult LDLT, IVC resection-interposition in a pediatric recipient is difficult, because a size-matched vein homograft should be prepared. Owing to the ongoing growth of the pediatric recipient, any prosthetic vessel graft cannot be used, particularly in infant patients. There have been only a few reports on IVC replacement in the field of pediatric LDLT. ${ }^{1-3}$

In our previously reported case of pediatric LDLT with IVC interposition, ${ }^{1}$ we had to wait for more than one month to obtain a cold-stored fresh IVC homograft, which was occasionally recovered from a deceased organ donor. In contrast, we could decide on the LDLT operation schedule promptly in the present case, because we already had a cold-preserved iliac vein homograft, which was also recovered from a deceased organ donor. In the current organ recovery setting in Korea, the iliac vein homografts have been more frequently retrieved than have IVC homografts been. Because the body size of the infant recipient

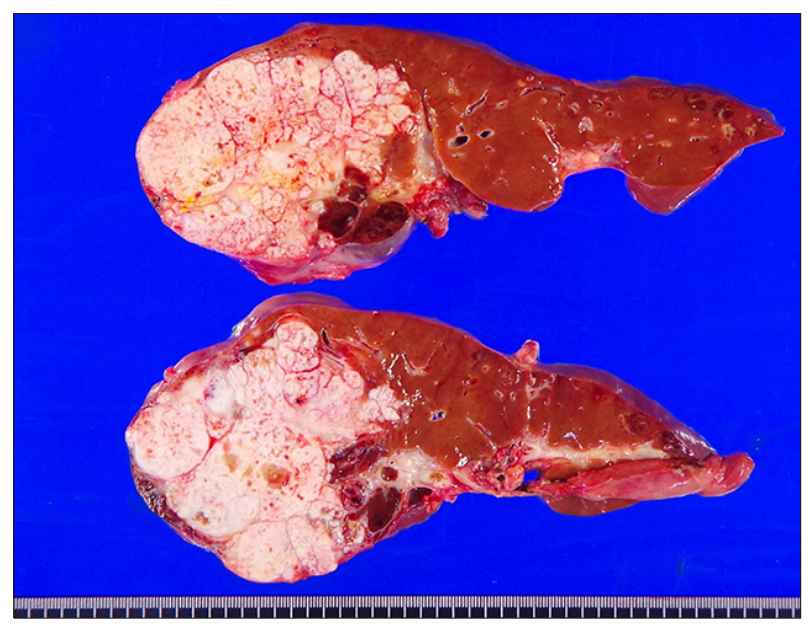

Fig. 5. Gross photographs of the explanted liver. There were multiple viable tumors with extensive necrosis. 

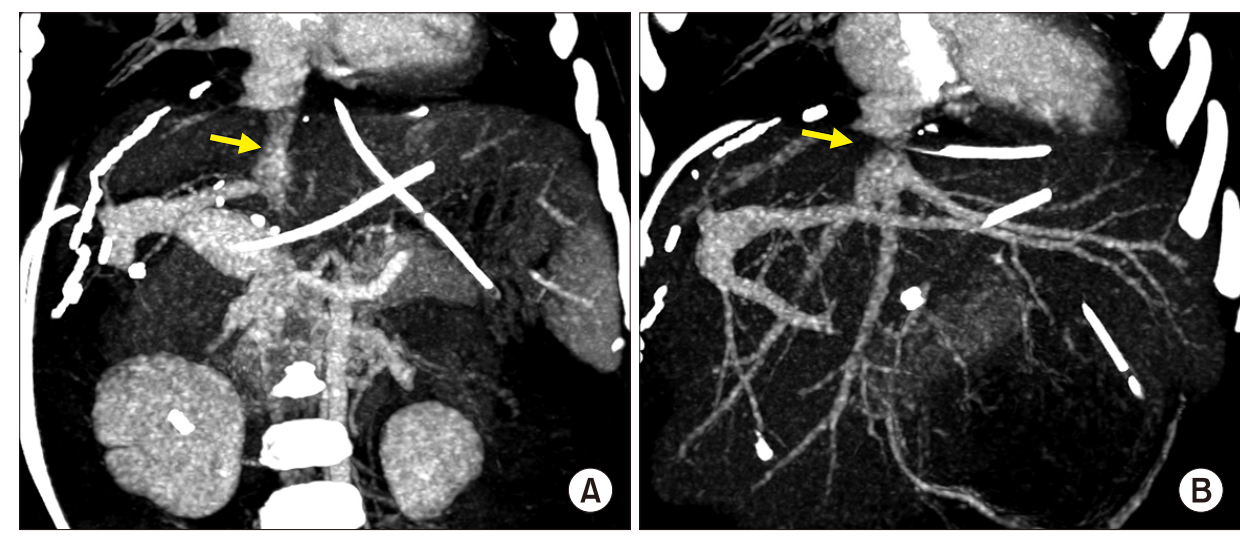

Fig. 6. Post-transplant computed tomography finding taken after five days. (A) The replaced retrohepatic inferior vena cava conduit is visualized (arrow). (B) The anastomosis site at the graft hepatic vein is visualized (arrow).
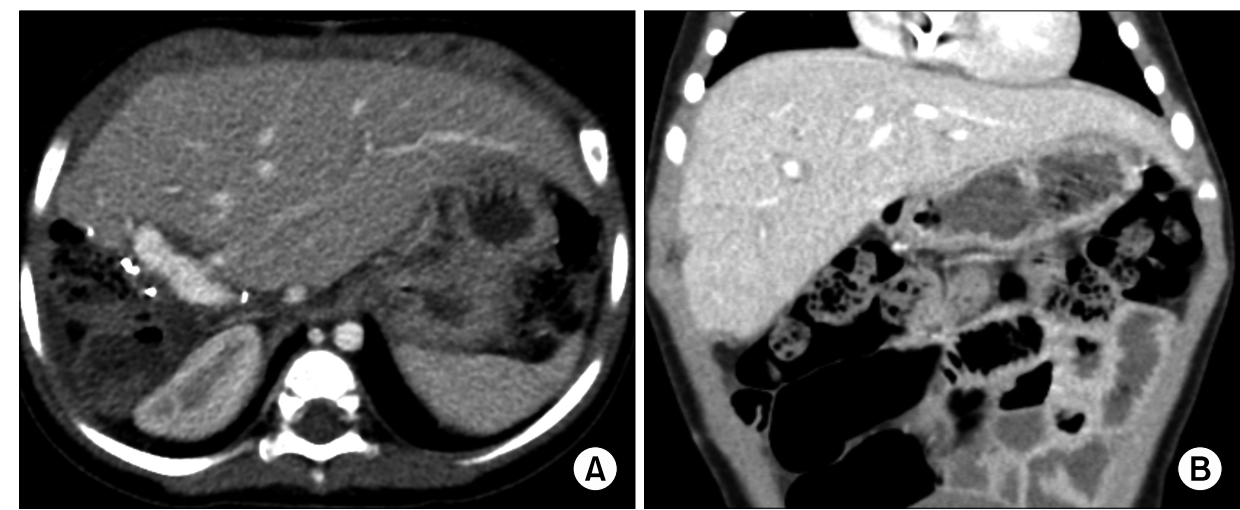

Fig. 7. Post-transplant computed tomography finding taken after 32 days. The normal-looking liver filled the abdomen without any vascular complication (A and $\mathrm{B}$ ). in this study was quite small, the size of the usual common iliac vein homograft was suitable for direct interposition.

During deceased organ donor surgery, sizable vessel homografts are retrieved after obtaining a written consent for tissue donation, which is registered in the tissue bank at our institution. To use a vessel homograft in a fresh state, it can be stored in a $4{ }^{\circ} \mathrm{C}$ Histidine-tryptophan-ketoglutarate (HTK) solution within seven days in principle instead by cryopreservation. The preservation period can be extended maximally to 14 days, but we observed a noticeable degradation of tissues as time passed. If not used within 14 days, they should be discarded, because considerable tissue damage had developed already during the 14-day preservation period, thus not allowing cryopreservation. ${ }^{4}$

In an in vitro study with saphenous vein samples observed up to 35 days, ${ }^{5}$ in terms of preserving the morphology of saphenous veins, phosphate-buffered saline and isotonic saline with heparin and antibiotic were the poorest, whereas Custodiol HTK and glutaraldehyde were the best. Demonstrating good retention of endothelial nitric oxide synthase staining throughout the study period, isotonic saline with heparin and antibiotic seems to have the best potential to retain vein wall functionality, despite relatively poor morphological preservation.

Although there are some studies favoring use of cryopreserved vessels in LDLT, $^{6-8}$ we have paid special attention to using a cryopreserved vein for portal vein or IVC interposition, since long-term patency of such vascular reconstruction is not fully evaluated yet. In a study on portal vein reconstruction in adult LDLT using cryopreserved vein grafts, ${ }^{9}$ the five-year primary and secondary patency rates of the cryopreserved vein grafts were $58 \%$ and $79 \%$, respectively.

In pediatric recipients, various vein homografts, such as iliac vein, IVC, and other large veins, can be used depending on the body size of the recipient and the availability of vessel grafts. We emphasize that cryopreserved veins should be avoided if possible for pediatric patients, because they can degenerate over time.

In conclusion, IVC replacement with a sizable vein homograft is a feasible option of LDLT to achieve complete tumor removal in pediatric patients with advanced hepato- 
blastoma.

\section{ORCID}

Jung-Man Namgoong: https://orcid.org/0000-0002-92377440

Shin Hwang: https://orcid.org/0000-0002-9045-2531

Seak Hee Oh: https://orcid.org/0000-0002-9672-8877

Kyung Mo Kim: https://orcid.org/0000-0001-7896-6751

Gil-Chun Park: https://orcid.org/0000-0003-1631-3258

Chul-Soo Ahn: https://orcid.org/0000-0002-3844-3646

Hyunhee Kwon: https://orcid.org/0000-0001-6647-9155

Yu Jeong Cho: https://orcid.org/0000-0001-6823-2746

Yong Jae Kwon: https://orcid.org/0000-0001-9490-1229

\section{AUTHOR CONTRIBUTIONS}

Conceptualization: SH, JMN.

Data curation: SHO, KMK.

Formal analysis: GCP, CSA, HK, YJC, YJK.

Funding acquisition: Not applicable.

Methodology: JMN, SH.

Project administration:SH.

Visualization: JMN, SH.

Writing - original draft: JMN, SH.

Writing - review \& editing: SH.

\section{REFERENCES}

1. Namgoong JM, Choi JU, Hwang S, Oh SH, Park GC. Pediatric living donor liver transplantation with homograft replacement of retrohepatic inferior vena cava for advanced hepatoblastoma. Ann Hepatobiliary Pancreat Surg 2019;23:178-182.

2. Sasaki K, Kasahara M, Fukuda A, Shigeta T, Tanaka H, Nakagawa $\mathrm{S}$, et al. Living donor liver transplantation with vena cava reconstruction using a cryopreserved allograft for a pediatric patient with Budd-Chiari syndrome. Transplantation 2009; 87:304-305.

3. Chardot C, Saint Martin C, Gilles A, Brichard B, Janssen M, Sokal E, et al. Living-related liver transplantation and vena cava reconstruction after total hepatectomy including the vena cava for hepatoblastoma. Transplantation 2002;73:90-92.

4. Hwang S, Kim DY, Ahn CS, Moon DB, Kim KM, Park GC, et al. Computational simulation-based vessel interposition reconstruction technique for portal vein hypoplasia in pediatric liver transplantation. Transplant Proc 2013;45:255-258.

5. Aavik A, Kibur RT, Lieberg J, Lepner U, Aunapuu M, Arend A. Cold-stored venous allografts in different preserving solutions: a study on changes in vein wall morphology. Scand J Surg 2019;108:67-75.

6. Aydin C, Ince V, Otan E, Akbulut S, Koc C, Kayaalp C, et al. Storage of allogeneic vascular grafts: experience from a high-volume liver transplant institute. Int Surg 2013;98:170-174.

7. Kim SH, Hwang S, Ha TY, Song GW, Jung DH, Ahn CS, et al. Usability of cryopreserved aortic allografts for middle hepatic vein reconstruction during living-donor liver transplantation. J Gastrointest Surg 2016;20:1049-1055.

8. Hwang S, Lee SG, Ahn CS, Park KM, Kim KH, Moon DB, et al. Cryopreserved iliac artery is indispensable interposition graft material for middle hepatic vein reconstruction of right liver grafts. Liver Transpl 2005;11:644-649.

9. Sugawara Y, Makuuchi M, Tamura S, Matsui Y, Kaneko J, Hasegawa $\mathrm{K}$, et al. Portal vein reconstruction in adult living donor liver transplantation using cryopreserved vein grafts. Liver Transpl 2006;12:1233-1236. 